\title{
Maybe repressed mRNAs are not stored in the chromatoid body in mammalian spermatids
}

\author{
Kenneth C Kleene and Danielle L Cullinane \\ Department of Biology, University of Massachusetts Boston, Boston, Massachusetts 02125-3393, USA \\ Correspondence should be addressed to KC Kleene; Email: kenneth.kleene@umb.edu
}

\begin{abstract}
The chromatoid body is a dynamic organelle that is thought to coordinate the cytoplasmic regulation of mRNA translation and degradation in mammalian spermatids. The chromatoid body is also postulated to function in repression of mRNA translation by sequestering dormant $m R N A s$ where they are inaccessible to the translational apparatus. This review finds no convincing evidence that dormant mRNAs are localized exclusively in the chromatoid body. This discrepancy can be explained by two hypotheses. First, experimental artifacts, possibly related to peculiarities of the structure and function of the chromatoid body, preclude obtaining an accurate indication of mRNA localization. Second, mRNA is not stored in the chromatoid body, because, like perinuclear $P$ granules in Caenorhabditis elegans, the chromatoid body functions as a center for mRNP remodeling and export to other cytoplasmic sites. Reproduction (2011) 142 383-388
\end{abstract}

\section{Introduction}

Post-transcriptional control of mRNA translation and degradation in the cytoplasm has important roles in controlling gene expression in mammalian spermatids. All mRNAs are partially translationally repressed in free mRNPs in meiotic and haploid spermatogenic cells, and some mRNAs are strongly repressed in early spermatids and recruited onto polysomes for translation in late spermatids (Kleene 2003). In addition, some mRNA species are degraded at specific steps of spermiogenesis, which ends the period that mRNAs can be translated to make protein (Heidaran et al. 1988, Mali et al. 1989, Saunders et al. 1992).

A series of recent articles propose that mRNA degradation and translational repression in spermatids are coordinated by the chromatoid body, an irregular, 1.0-1.5 $\mu \mathrm{M}$ diameter sponge-like organelle, which is not surrounded by a membrane (reviewed in Parvinen (2005) and Kotaja \& Sassone-Corsi (2007)). The chromatoid body is divided into an electron-dense fibrous stroma and less-dense lacunae. During spermiogenesis, the chromatoid body undergoes dynamic changes in shape, position, and size. The position of the chromatoid body close to nuclear pores in early spermatids is thought to facilitate transfer of RNA to the chromatoid body. A role of the chromatoid body in post-transcriptional gene regulation is inferred from evidence that it contains factors that mediate mRNA degradation and translational repression such as miRNAs, piRNAs, the XRN1 RNase, decapping enzymes, argonaute proteins,
RNA helicases, and Y-box protein MSY2 (Kotaja \& Sassone-Corsi 2007). The functional importance of the chromatoid body is demonstrated by findings that knockouts of genes encoding constituents of the chromatoid body produce blocks to spermiogenesis, male infertility, and occasional failure to activate translation in late spermatids (Deng \& Lin 2002, Tsai-Morris et al. 2004, Vasileva et al. 2009, Yabuta et al. 2011). The caveat should be noted that it is very difficult to assign specific functions from knockouts of genes that impact many cellular processes.

The chromatoid body is an RNA granule, a class of organelles that is popularly thought to sequester dormant mRNAs for degradation and storage. RNA granules come in a variety of types, processing bodies (P-bodies), stress granules, germ cell granules, and neuronal granules (Anderson \& Kedersha 2009). RNA granules in germ cells exhibit a characteristic fibrous structure and are referred to as nuage (Parvinen 2005, Seydoux \& Braun 2006, Anderson \& Kedersha 2009). The various types of granules differ in size, cellular location, physiological and developmental cues that promote their formation and disappearance and content of specific factors and mRNAs. In the simplest situation, translationally inactive mRNAs are localized in RNA granules and translationally active mRNAs are distributed throughout the cytoplasm on polysomes, but more complicated models exist in which inactive mRNAs are transferred from one type of RNA granule to another (Anderson \& Kedersha 2009, Sheth et al. 2010). 
The best-characterized RNA granules, P-bodies and stress granules, are dynamic. Many observations are explained by simple rules: conditions that increase the levels of dormant mRNAs increase the size and number of P-bodies and stress granules and conditions that decrease the levels of dormant mRNAs decrease the mass of RNA granules (Anderson \& Kedersha 2009, Balagopal \& Parker 2009). The formation of P-bodies and stress granules that are visible under the light microscope is mediated by concentration-dependent aggregation of prion-like domains of proteins associated with dormant mRNAs (Gilks et al. 2004, Decker et al. 2007). However, mutations, which abrogate the formation of stress granules and P-bodies, do not inactivate translational repression and mRNA degradation demonstrating that visible granules are not required for these processes (Eulalio et al. 2007, Anderson \& Kedersha 2009, Balagopal \& Parker 2009).

The dynamic nature of stress granules is further demonstrated by fluorescence recovery after photobleaching (Kedersha et al. 2005). A high intensity flash of laser light was used to bleach fluorescent proteins fused to a variety of stress granule factors, which were replenished in stress granules by migration from the surrounding cytoplasm in 30S. Presumably, the rapid import of factors into stress granules is balanced by rapid export because stress granules do not continuously increase in size.

Several groups favor the idea that newly synthesized mRNAs are transferred directly from the nucleus to the chromatoid body, where they are stored initially as translationally repressed free mRNPs, later exiting for translation on polysomes (Kotaja \& Sassone-Corsi 2007, Nguyen Chi et al. 2009, Tsai-Morris et al. 2010). Sequestration in the chromatoid body has also been proposed as a mechanism of repression of mRNA translation in spermatids. The literature reviewed below supports the idea that substantial levels of dormant mRNA are present in the general cytoplasm in spermatids. The purpose of this review is to describe and interpret these observations.

\section{Cytological and biochemical studies of mRNA localization in the chromatoid body}

Evidence that translationally repressed mRNA is stored in the chromatoid body is primarily derived from a single study of the transition protein 2 (Tnp2) mRNA in rat testis (Saunders et al. 1992). The Tnp2 mRNA is expressed in step 7-12 spermatids, and is translationally repressed in steps 7 and 8 and translationally active starting in step 9 (Kistler et al. 1996). Saunders et al. (1992) observed that the Tnp2 mRNA is strongly localized adjacent to the nucleus in step 7 using digoxigenin-based non-isotopic in situ hybridization, fixation by perfusion with Bouin's, and $2 \mu \mathrm{m}$ polystyrene sections. Although immunological markers, which would reliably identify the chromatoid body, were not available at this time, the size and perinuclear location of the hybridization signal are consistent with the chromatoid body. However, the photographs also show less intense in situ hybridization signal throughout the cytoplasm and lack of localization in $5 \mu \mathrm{m}$ paraffin sections of step 7 spermatids. The hybridization signal in step 8-12 spermatids is stronger and unlocalized. Although it is difficult to assess by eye the proportions of localized and unlocalized Tnp2 mRNA in step 7 spermatids, the possibility merits consideration that unlocalized mRNA predominates, because the chromatoid body occupies $\sim 0.4 \%$ of the cytoplasmic volume, based on the relative diameters of the chromatoid body, $\sim 1.5 \mu \mathrm{m}$, and round spermatid cells and nuclei, 10 and $5 \mu \mathrm{m}$ (Romrell et al. 1976, Parvinen 2005).

It would be reasonable to expect that the literature would contain many reports of mRNA localization in the chromatoid body, because the developmental expression of many mRNAs in spermiogenesis has been analyzed with in situ hybridization. Most mRNAs should exhibit localization because each of more than 50 mRNA species that are expressed in spermatids that have been analyzed with sucrose gradients exhibit high levels of translationally inactive free mRNPs, usually $>50 \%$ (Kleene 2003). However, useful information is limited to a relatively small group of studies that utilize non-isotopic hybridization and good fixation and present photographs in which the location of the hybridization signal can be visualized. All of these studies show no localization (Weitzel et al. 2003, lida et al. 2004, Ellis et al. 2005, Kogami et al. 2006). In contrast, Fukuda et al. (2004) reported that the MOR23 mRNA is localized close to the nucleus (chromatoid body?), and that the histone $\mathrm{H} 1 \mathrm{t}$ mRNA is associated with an elongated perinuclear structure in pachytene spermatocytes, which resembles the chromatoid body in meiotic cells (Parvinen 2005). This study also did not report localization of the Prm1 mRNA and does not meet the technical standards mentioned above.

Morales et al. (1991) argue that the Prm1 and Tnp1 mRNAs are not localized in the chromatoid body. In these studies, the testes were fixed by perfusion with $2 \%$ glutaraldehyde and $4 \%$ paraformaldehyde, embedded in agar, and sectioned at $100 \mu \mathrm{m}$. These thick sections were hybridized to antisense ${ }^{3} \mathrm{H}$-riboprobes followed by washes, osmium staining, embedding in epon, thin sectioning and light and electron microscope autoradiography. These preparations beautifully preserve the ultrastructure of the chromatoid body, and reveal that the Prm1 and Tnp1 mRNAs are uniformly distributed in the cytoplasm of step 7-9 spermatids.

Unfortunately, the use of glutaraldehyde in these experiments is problematic. Lawrence \& Singer (1985) demonstrated that glutaraldehyde increases the background and sharply decreases the specific in situ 
hybridization signal because it cross-links cytoplasmic proteins tightly, rendering mRNA inaccessible to the hybridization probe and decreasing the efficiency of removal of non-hybridized probe by the washes. These problems would likely be aggravated by performing in situ hybridization on thick sections. This appears to be a problem because the Prm1 and Tnp1 in situ hybridization signals are present over the nuclei and cytoplasm of pachytene spermatocytes and step 1-6 spermatids, which conflicts with many studies demonstrating that these mRNAs are first detected in step 7 spermatids (Heidaran et al. 1988, Braun et al. 1989, Mali et al. 1989). The absence of hybridization signals in late spermatids and with sense-strand negative controls may reflect low penetration of the probes deep into the tissue.

Recent studies have demonstrated the localization of RNAs and proteins in the chromatoid body with dried-down preparations (Kotaja et al. 2006), in which suspensions of dispersed cells from short pieces of seminiferous tubules are added to dilute Triton X-100 and paraformaldehyde and the cells are dried on microscope slides. Unfortunately, dried-down preparations cannot be used to analyze the levels of proteins and RNAs in the cytoplasm, because phase contrast images show nuclei and perinuclear chromatoid bodies, but the cytoplasm is invisible because most of it is lost. The loss of cytoplasm is further supported by findings that several factors (poly(A), DICER, AGO3, and DCP1A) are present in both the chromatoid body and cytoplasm in squash preparations which retain the cytoplasm, but are undetectable in the area surrounding the nuclei and chromatoid body in dried-down preparations (Kotaja et al. 2006). Kotaja et al. (2006) did not report the presence of specific mRNAs in the chromatoid body in dried-down preparations. The loss of cytoplasm also raises the question whether RNAs and proteins are retained completely by the chromatoid body and nuclei in dried-down preparations.

Nguyen Chi et al. (2009) reported that the Gonf and $\mathrm{Brd} 2 \mathrm{mRNAs}$ are localized in the chromatoid body in dried-down preparations of early spermatids. Again, it is unclear what proportion of each mRNA is in the general cytoplasm.

Not only is the localization of translationally repressed mRNA in the chromatoid body poorly documented, but factors that are associated with translationally repressed mRNAs also are not exclusively associated with the chromatoid body. The most convincing studies concern mouse Y-box proteins, MSY2, and MSY4, because western blot analyses of sucrose gradients demonstrate that both proteins sediment exclusively with free mRNPs with virtually no free protein sedimenting at the top of the gradient (Davies et al. 2000, Giorgini et al. 2002, Yang et al. 2005). Light microscope immunocytochemistry of paraffin sections of adult testis with anti-FRGY2, the Xenopus laevis homolog of MSY2, and anti-MSY4 reveals that both proteins are distributed throughout the cytoplasm (Oko et al. 1996, Davies et al. 2000). In contrast, electron microscope immunocytochemistry demonstrates that MSY2 is concentrated in the lacunae and immediate vicinity of the chromatoid body, and at lower levels throughout the general cytoplasm. Presumably, the strong light microscope MSY2 signal in the cytoplasm obscures slightly higher levels in the chromatoid body.

The localization of other mRNA-binding proteins is more difficult to interpret. For example, western blot analysis of sucrose gradients demonstrates that the vast majority of cytoplasmic poly(A)-binding protein PABPC2 sediments more slowly than single ribosomes (Kimura et al. 2009), but PABPC2 is present as free protein at the top of the gradient and free mRNPs sedimenting with a peak at about 60S. This complicates interpretation of immunocytochemical detection of PABPC2 in the chromatoid body and the general cytoplasm, because it is unclear whether the localized and unlocalized PABPC2 correspond to free mRNPs or free protein.

Cell fractionation yields another striking contradiction with the idea that translationally inactive free mRNPs are sequestered in the chromatoid body. The chromatoid body is a rather large structure, which pellets during centrifugation at 500-1000 $\mathrm{g}$ for $10 \mathrm{~min}$ (Figueroa \& Burzio 1998, Meikar et al. 2010). Thus, the chromatoid body would be expected to sediment with nuclei in preparing cytoplasmic extracts for sucrose gradient analysis, $13000 \mathrm{~g}$ for $2 \mathrm{~min}$. However, using two different methods of RNA extraction, $8.8 \pm 4.3 \%$ (mean and S.D. of four experiments) of the Smcp mRNA pellets with nuclei in adult testis (KC Kleene, unpublished observations), whereas $\sim 65 \%$ sediments as free mRNPs near the top of the sucrose gradients after centrifugation at $125000 \mathrm{~g}$ for $80 \mathrm{~min}$ (Bagarova et al. 2010). These findings suggest either that Smcp free mRNPs are not localized in the chromatoid body in intact cells or that free mRNPs are released into the cytoplasm during cell fractionation. Studies in yeast also indicate that repressed mRNAs in P-bodies in intact cells sediment as free mRNPs in sucrose gradients (Berengues et al. 2005). P-bodies and stress granules are remarkably fragile and these organelles have never been isolated.

Meikar et al. (2010) purified the chromatoid body from paraformaldehyde-fixed cells from adult testis with differential centrifugation and immunoprecipitation with antibody to DEAD (Asp-Glu-Ala-Asp) box polypeptide 4 (DDX4; also known as mouse vasa homolog, $\mathrm{MVH}$ ), a marker for the chromatoid body. After reversal of the cross-linking and RNA extraction, the levels of Odf1, Prm1, and Tnp2 mRNAs were analyzed in all fractions with RT-PCR. Sucrose gradient analysis demonstrates that $\sim 75 \%$ of all three mRNAs are present in free mRNPs in adult testis and purified elongated spermatids (Kleene 1989, Cataldo et al. 1999), all of which would be expected to copurify with the chromatoid body if it stores dormant mRNAs for later translation. In contrast, 
Meikar et al. (2010) observed that the vast majority of all three mRNAs are in the supernatant from the initial centrifugation, and that negligible amounts are present in the initial and anti-DDX4 pellets. The high levels of these mRNAs in the supernatant may represent polysomal mRNAs and free mRNPs in the general cytoplasm or free mRNPs that exit the chromatoid body after cell lysis, but the very small fraction of free mRNPs that copurifies with the chromatoid body does not support the idea that the free mRNPs are stored in that organelle.

\section{Summary and perspective}

The information surveyed above contains little convincing evidence that translationally inactive mRNAs are localized in chromatoid body in mammalian spermatids. Indeed, the studies that are usually cited as evidence for mRNA localization in the chromatoid body demonstrate significant levels in the general cytoplasm (Saunders et al. 1992, Oko et al. 1996). As discussed below, this may be either an experimental artifact or an important insight into the function of the chromatoid body.

The failure to detect strong localization of translationally repressed mRNA in the chromatoid body in whole cells could be caused by two artifacts: mRNAs in free mRNPs in the chromatoid body may be masked by protein and inaccessible to hybridization probes, and free mRNPs may leak out of the chromatoid body before the fixative immobilizes the mRNPs. Both artifacts are plausible considering that repressed mRNAs may be coated with protein, and the disaggregation of P-bodies in sucrose gradients and the rapid exchange of factors between stress granules and the cytoplasm. A rigorous approach would be to analyze the localization of free mRNPs in living cells with tethering assays by coexpressing an mRNA containing a 3'-UTR-binding site for the bacteriophage MS2 coat protein and an mRNA encoding a GFP-MS2 coat protein fusion in prepubertal transgenic mice (Sheth \& Parker 2003, Kedersha et al. 2005). However, tethering assays require optimization of the relative levels of target mRNA and GFP coat protein to visualize localization.

The alternative idea that high levels of mRNA are not stored in the chromatoid body is consistent with work on Caenorhabditis elegans demonstrating that there are multiple types of $\mathrm{P}$ granules (Schisa et al. 2001, Noble et al. 2008, Sheth et al. 2010). One class is associated with nuclear pore complexes, as are chromatoid bodies in early spermatids. These perinuclear $\mathrm{P}$ granules function as mRNP remodeling and sorting centers: newly synthesized mRNAs pass through the $\mathrm{P}$ granules and are exported to the cytoplasm or other classes of cytoplasmic P granules. Indeed, the observation that a number of constituents of the chromatoid body are also present in the cytoplasm (DICER, AGO3, DCP1A, and MIWI; Grivna et al. 2006, Kotaja et al. 2006) implies that these factors have functions in both the general cytoplasm and the chromatoid body. It is relevant to note that DEAD-box helicases, a major constituent of the chromatoid body and other forms of nuage, often have functions in melting RNA secondary structure that promote the formation of protein-mRNA complexes (Arkov \& Ramos 2010). In addition, the higher concentration of factors in the chromatoid body than that in the general cytoplasm may promote the association of factors with mRNAs in transit.

The hypothesis that the chromatoid body functions in mRNP remodeling and export raises several questions. Are repressed mRNAs in spermatids stored as individual free mRNPs or aggregates of free mRNPs? The chromatoid body is the only type of nuage that is visible in the electron microscope in early spermatids (Eddy 1974, Parvinen 2005). However, free mRNPs might be associated with constituents of the chromatoid body which are dispersed throughout the cytoplasm in spermatids, and it is possible that some repressed mRNAs are stored in the chromatoid body and others are not.

Other questions concern the mechanisms that distinguish mRNAs that are strongly repressed in early spermatids (e.g. Prm1, Tnp2, and Smcp) from mRNAs that are partially active and partially repressed (e.g. $L d h c$ and Pgk2). Work in C. elegans demonstrates that $3^{\prime}$-UTR elements direct specific mRNAs to different classes of granules thereby controlling both temporal and spatial expression (Noble et al. 2008). However, in the absence of evidence for RNA granules that harbor dormant mRNAs in spermatids, the elements in the Prm1 and Smcp 3'-UTR, which control the timing of translation in spermatids, may act purely by producing free mRNPs, likely by a variant of the closed-loop model (Giorgini et al. 2001, Zhong et al. 2001, Bagarova et al. 2010, Jackson et al. 2010). This line of reasoning also suggests that much could be learned by studying factors that have been implicated in post-transcriptional regulation and nuage in real model systems, flies and worms.

\section{Declaration of interest}

The authors declare that there is no conflict of interest that could be perceived as prejudicing the impartiality of this review.

\section{Funding}

This review was supported by grant MCB-0642128 from the National Science Foundation.

\section{References}

Anderson P \& Kedersha N 2009 RNA granules: post-transcriptional and epigenetic modulators of gene expression. Nature Reviews. Molecular Cell Biology 10 430-436. (doi:10.1038/nrm2694) 
Arkov AL \& Ramos A 2010 Building RNA-protein granules: insight from the germline. Trends in Cell Biology 20 482-490. (doi:10.1016/j.tcb.2010. 05.004)

Bagarova J, Chowdhury TA, Kimura M \& Kleene KC 2010 Identification of elements in the Smcp $5^{\prime}$ and $3^{\prime}$ UTR that repress translation and promote the formation of heavy inactive mRNPs in spermatids by analysis of mutations in transgenic mice. Reproduction 140 853-864. (doi:10.1530/ REP-10-0323)

Balagopal V \& Parker R 2009 Polysomes, P bodies and stress granules, states and fates of eukaryotic mRNAs. Current Opinion in Cell Biology 21 403-408. (doi:10.1016/j.ceb.2009.03.005)

Berengues M, Teixeira D \& Parker R 2005 Movement of eukaryotic mRNAs between polysomes and cytoplasmic processing bodies. Science $\mathbf{3 1 0}$ 486-489. (doi:10.1126/science.1115791)

Braun RE, Peschon JJ, Behringer RR, Brinster RL \& Palmiter RD 1989 Protamine $3^{\prime}$-untranslated sequences regulate temporal translational control and subcellular localization of growth hormone in spermatids of transgenic mice. Genes and Development 3 793-802. (doi:10.1101/ gad.3.6.793)

Cataldo L, Mastrangelo MA \& Kleene KC 1999 A quantitative sucrose gradient analysis of the translational activity of 18 mRNA species in testes from adult mice. Molecular Human Reproduction 5 206-213. (doi:10.1093/molehr/5.3.206)

Davies HG, Giorgini F, Fajardo MA \& Braun RE 2000 A sequence-specific RNA binding complex expressed in murine germ cells contains MSY2 and MSY4. Developmental Biology 221 87-100. (doi:10.1006/dbio. 2000.9658)

Decker CJ, Teixeira D \& Parker R 2007 Edc3p and a glutamine/ asparagine-rich domain of $\mathrm{Lsm} 4 \mathrm{p}$ function in processing body assembly in Saccharomyces cerevisiae. Journal of Cell Biology $\mathbf{1 7 9}$ 437-449. (doi:10.1083/jcb.200704147)

Deng W \& Lin H 2002 Miwi, a murine homolog of piwi, encodes a cytoplasmic protein essential for spermatogenesis. Developmental Cel/ 2 819-830. (doi:10.1016/S1534-5807(02)00165-X)

Eddy EM 1974 Fine structural observations on the form and distribution of nuage in germ cells of the rat. Anatomical Record 178 731-757. (doi:10.1002/ar.1091780406)

Ellis PJ, Clemente EJ, Ball P, Touré A, Ferguson L, Turner JM, Loveland KL, Affara NA \& Burgoyne PS 2005 Deletions on mouse Yq lead to upregulation of multiple X-and Y-linked transcripts in spermatids. Human Molecular Genetics 14 2705-2715. (doi:10.1093/hmg/ddi304)

Eulalio A, Behm-Ansmant I, Schweizer D \& Izaurralde E 2007 P-body formation is a consequence, not the cause, of RNA-mediated gene silencing. Molecular and Cellular Biology 27 3970-3981. (doi:10.1128/ MCB.00128-07)

Figueroa J \& Burzio LO 1998 Polysome-like structures in the chromatoid body of rat spermatids. Cell Tissue Research 291 575-579. (doi:10.1007/ s004410051027)

Fukuda N, Yomogida K, Okabe M \& Touhara K 2004 Functional characterization of a mouse testicular olfactory receptor and its role in chemosensing and in regulation of sperm motility. Journal of Cell Science 117 5835-5845. (doi:10.1242/jcs.01507)

Gilks N, Kedersha N, Ayodele M, Shen L, Stoecklin G, Dember LM \& Anderson P 2004 Stress granule assembly is mediated by prion-like aggregation of TIA-1. Molecular Biology of the Cell 15 5383-5398. (doi:10.1091/mbc.E04-08-0715)

Giorgini F, Davies HG \& Braun RE 2001 MSY2 and MSY4 bind a conserved sequence in the $3^{\prime}$-untranslated region of protamine $1 \mathrm{mRNA}$ in vitro and in vivo. Molecular and Cellular Biology 21 7010-7019. (doi:10. 1128/MCB.21.20.7010-7019.2001)

Giorgini F, Davies HG \& Braun RE 2002 Translational repression by MSY4 inhibits spermatid differentiation in mice. Development $\mathbf{1 2 9}$ 3669-3679.

Grivna ST, Pyhtila B \& Lin H 2006 MIWI associates with translational machinery and PIWI-interacting RNAs (piRNAs) in regulating spermatogenesis. PNAS 103 13415-13420. (doi:10.1073/pnas.0605506103)

Heidaran MA, Showman RM \& Kistler WS 1988 A cytochemical study of the transcriptional and translational regulation of nuclear transition protein 1 (TP1), a major chromosomal protein of mammalian spermatids. Journal of Cell Biology 106 1427-1433. (doi:10.1083/jcb.106.5.1427) lida H, Yamashita H, Doiguchi M \& Kaneko T 2004 Molecular cloning of rat Spergen-3, a spermatogenic cell-specific gene-3, encoding a novel $75 \mathrm{kDa}$ protein bearing EF-hand motifs. Journal of Andrology 25 885-892.

Jackson RJ, Hellen CU \& Pestova TV 2010 The mechanism of eukaryotic translation initiation and principles of its regulation. Nature Reviews. Molecular Cell Biology 11 113-127. (doi:10.1038/nrm2838)

Kedersha N, Stoecklin G, Ayodele M, Yacono P, Lykke-Andersen J, Fritzler MJ, Scheuner D, Kaufman RJ, Golan DE \& Anderson P 2005 Stress granules and processing bodies are dynamically linked sites of mRNP remodeling. Journal of Cell Biology 169 871-884. (doi:10.1083/ jcb.200502088)

Kimura M, Ishida K, Kashiwabara S \& Baba T 2009 Characterization of two cytoplasmic poly(A)-binding proteins, PABPC1 and PABPC2, in mouse spermatogenic cells. Biology of Reproduction 80 545-554. (doi:10.1095/biolreprod.108.072553)

Kistler WS, Henriksén K, Mali P \& Parvinen M 1996 Sequential expression of nucleoproteins during rat spermiogenesis. Experimental Cell Research 225 374-381. (doi:10.1006/excr.1996.0188)

Kleene KC 1989 Poly(A), shortening accompanies the activation of translation of five mRNAs during spermiogenesis in the mouse. Development 106 367-373.

Kleene KC 2003 Patterns, mechanisms, and functions of translation regulation in mammalian spermatogenic cells. Cytogenetic and Genome Research 103 217-224. (doi:10.1159/000076807)

Kogami T, Miki Y, Yamada T, Umegaki T, Nishimura M, Amo T, Kosaka J \& Sasaki J 2006 Quantification of PERF15 mRNA in tissue sections from rat testes. Acta Histochemica et Cytochemica 39 183-192. (doi:10.1267/ ahc.06016)

Kotaja N \& Sassone-Corsi P 2007 The chromatoid body: a germ-cellspecific RNA-processing centre. Nature Reviews. Molecular Cell Biology 8 85-90. (doi:10.1038/nrm2081)

Kotaja N, Bhattacharyya SN, Jaskiewicz L, Kimmins S, Parvinen $M$, Filipowicz W \& Sassone-Corsi P 2006 The chromatoid body of male germ cells, similarity with processing bodies and presence of Dicer and microRNA pathway components. PNAS 103 2647-2652. (doi:10.1073/ pnas.0509333103)

Lawrence JB \& Singer RH 1985 Quantitative analysis of in situ hybridization methods for the detection of actin gene expression. Nucleic Acids Research 13 1777-1799. (doi:10.1093/nar/13.5.1777)

Mali P, Kaipia A, Kangasniemi M, Toppari J, Sandberg M, Hecht NB \& Parvinen M 1989 Stage-specific expression of nucleoprotein mRNAs during rat and mouse spermiogenesis. Reproduction, Fertility, and Development 1 369-382. (doi:10.1071/RD9890369)

Meikar O, Da Ros M, Liljenbäck H, Toppari J \& Kotaja N 2010 Accumulation of piRNAs in the chromatoid bodies purified by a novel isolation protocol. Experimental Cell Research 316 1567-1575. (doi:10. 1016/j.yexcr.2010.02.023)

Morales CR, Kwon YK \& Hecht NB 1991 Cytoplasmic localization during storage and translation of the mRNAs of transition protein 1 and protamine 1 , two translationally regulated transcripts of the mammalian testis. Journal of Cell Science 100 119-131.

Nguyen Chi M, Chalmel F, Agius E, Vanzo N, Khabar KS, Jégou B \& Morello D 2009 Temporally regulated traffic of HuR and its associated ARE-containing mRNAs from the chromatoid body to polysomes during mouse spermatogenesis. PLOS ONE 4 e4900. (doi:10.1371/journal.pone. 0004900)

Noble SL, Allen BL, Goh LK, Nordick K \& Evans TC 2008 Maternal mRNAs are regulated by diverse $\mathrm{P}$ body-related mRNP granules during early Caenorhabditis elegans development. Journal of Cell Biology 182 559-572. (doi:10.1083/jcb.200802128)

Oko R, Korley R, Murray MT, Hecht NB \& Hermo L 1996 Germ cellspecific DNA and RNA binding proteins p48/52 are expressed at specific stages of male germ cell development and are present in the chromatoid body. Molecular Reproduction and Development $\mathbf{4 4}$ 1-13. (doi:10.1002/(SICI)1098-2795(199605)44:1 < 1::AID-MRD1 > 3.0. CO; $2-\mathrm{S})$

Parvinen M 2005 The chromatoid body in spermatogenesis. International Journal of Andrology 28 189-201. (doi:10.1111/j.1365-2605.2005. 00542.x) 
Romrell LJ, Bellvé AR \& Fawcett DW 1976 Separation of mouse spermatogenic cells by sedimentation velocity. A morphological characterization. Developmental Biology 49 119-131. (doi:10.1016/ 0012-1606(76)90262-1)

Saunders PT, Millar MR, Maguire SM \& Sharpe RM 1992 Stage-specific expression of rat transition protein 2 mRNA and possible localization to the chromatoid body of step 7 spermatids by in situ hybridization using a nonradioactive riboprobe. Molecular Reproduction and Development 33 385-391. (doi:10.1002/mrd.1080330404)

Schisa JA, Pitt JN \& Priess JR 2001 Analysis of RNA associated with P granules in germ cells of C. elegans adults. Development 128 1287-1298.

Seydoux G \& Braun RE 2006 Pathway to totipotency, lessons from germ cells. Cell 127 891-904. (doi:10.1016/j.cell.2006.11.016)

Sheth U \& Parker R 2003 Decapping and decay of messenger RNA occur in cytoplasmic processing bodies. Science 300 805-808. (doi:10.1126/ science.1082320)

Sheth U, Pitt J, Dennis S \& Priess JR 2010 Perinuclear P granules are the principal sites of mRNA export in adult C. elegans germ cells. Development 137 1305-1314. (doi:10.1242/dev.044255)

Tsai-Morris CH, Sheng Y, Lee E, Lei KJ \& Dufau ML 2004 Gonadotropinregulated testicular RNA helicase $(G R T H / D d x 25)$ is essential for spermatid development and completion of spermatogenesis. PNAS 101 6373-6378. (doi:10.1073/pnas.0401855101)

Tsai-Morris CH, Sheng Y, Gutti RK, Tang PZ \& Dufau ML 2010 Gonadotropin-regulated testicular RNA helicase (GRTH/DDX25): a multifunctional protein essential for spermatogenesis. Journal of Andrology 31 45-52. (doi:10.2164/jandrol.109.008219)
Vasileva A, Tiedau D, Firooznia A, Müller-Reichert T \& Jessberger R 2009 Tdrd6 is required for spermiogenesis, chromatoid body architecture, and regulation of miRNA expression. Current Biology 19 630-639. (doi:10. 1016/j.cub.2009.02.047)

Weitzel JM, Shiryaeva NB, Middendorff R, Balvers M, Radtke C, Ivell R \& Seitz HJ 2003 Testis-specific expression of rat mitochondrial glycerol-3-phosphate dehydrogenase in haploid male germ cells. Biology of Reproduction 68 699-707. (doi:10.1095/biolreprod.102. 008540)

Yabuta Y, Ohta H, Abe T, Kurimoto K, Chuma S \& Saitou M 2011 TDRD5 is required for retrotransposon silencing, chromatoid body assembly, and spermiogenesis in mice. Journal of Cell Biology 192 781-795. (doi:10. 1083/jcb.201009043)

Yang J, Medvedev S, Reddi PP, Schultz RM \& Hecht NB 2005 The DNA/RNA-binding protein MSY2 marks specific transcripts for cytoplasmic storage in mouse male germ cells. PNAS 102 1513-1518. (doi:10.1073/pnas.0404685102)

Zhong J, Peters AH, Kafer K \& Braun RE 2001 A highly conserved sequence essential for translational repression of the protamine 1 messenger RNA in murine spermatids. Biology of Reproduction 64 1784-1789. (doi:10. 1095/biolreprod64.6.1784)

Received 11 April 2011

First decision 17 May 2011

Accepted 14 June 2011 